\title{
Treatment outcomes of gall bladder cancer, IOyears experience
}

\begin{abstract}
Background: Gallbladder cancer (GBC) represents the most common and aggressive biliary tract cancer with overall 5-year survival being only 5\%-10\%. Survival following resection, especially in early stages, has shown some improvement due to advances in surgical treatment. Advanced stages, however, continue to have dismal outcome. Complete surgical resection offers the only chance for cure, which extended from simple cholecystectomy to major hepatectomy or enbloc resection of adjacent organs. The aim of this study was to examine the predictors of longterm survival in patients with gall bladder cancer.
\end{abstract}

Methods: We retrospectively evaluated 38 patients who underwent curative (R0) resection for GBC between January 2001 and December 2010 in Surgical Oncology Department, South Egypt Cancer Institute, Assiut University, Egypt. Surgical procedures included simple cholecystectomy $(10.5 \%)$, radical cholecystectomy $(75.4 \%)$, bile duct resection (33.8), and right hepatectomy (7.8\%). Adjacent organ resection was performed in $(23.4 \%)$, duodenal sleeve resection (10.4\%), segmental colectomy $(5.2 \%)$, segmental gastrectomy $(2.6 \%)$, Hepatopancreatico-duodenectomy (5.3\%). Adjuvant chemotherapy was given for $(68.4 \%)$ of patients and adjuvant radiotherapy for $(36.8 \%)$ of patients. Median follow up period of the patients was 38 months (0.5-69 months).

Results: The median disease free survival (DFS) for GBC patients was 38 months. Univariate analysis revealed that patient's age, comorbidities, weight loss, jaundice, tumor differentiation, organ invasion, lymph node metastasis, perineural invasion, tumor stage and chemotherapy were associated with the patient's survival. Of these, weight loss, jaundice, lymph node metastasis and tumor stage were found to influence the overall survival on the multivariate Cox Hazard Regression analysis. First year overall survival estimate was $86.84 \% \pm 5.5 \%, 2$ nd year $=71.05 \% \pm 7.4 \%$, Third year $60.53 \% \pm 7.9 \%$ fifth year $=15.79 \%$ $\pm 5.9 \%$.

Conclusion: Curative surgical resection remains the only effective approach for treatment of GBC. This study confirms that jaundice, weight loss and aggressive tumor (advanced stage and regional lymph nodes metastasis) are predictors of poor prognosis.

Keywords: cholecystectomy, gallbladder neoplasms, hepatectomy, prognosis, retrospective studies
Volume 3 Issue 4 - 2015

\author{
Ali Zedan,' Hussein Fakhry,' Murad Jabir,' \\ Abeer lbrahim $^{2}$ \\ 'Department of Surgical Oncology, Assiut University, Egypt \\ ${ }^{2}$ Department of Medical Oncology and Hematological \\ Malignancy, Assiut University, Egypt
}

Correspondence: Hussein Fakhry, Department of Surgical Oncology, South Egypt Cancer Institute, Assiut University, Assiut 7I I I I, Egypt, Tel 2.01064E+ I I, Fax 20882086609,

Email Hussein_hozayen@yahoo.com

Received: August 24, 2015 | Published: November 06, 2015
Abbreviations: GBC, gallbladder cancer; SECI, South Egypt cancer institute; $\mathrm{CT}$, computed tomography; MRI, magnetic resonance imaging; MRCP, magnetic resonance cholangiopancreatography; AJCC, American joint committee on cancer; SC, simple cholecystectomy; ELR, extended liver resection; HPD, hepatopancreatico-duodenectomy; RC, radical cholycystectomy; BD, bile duct resection; DVT, deep venous thrombosis

\section{Introduction}

Gallbladder cancer (GBC) is the most common malignancy of the biliary tract and the fifth cancer of the gastrointestinal tract that is frequently diagnosed at an advanced stage due to non specific symptoms, anatomic location and aggressive biologic behavior, with a poor prognosis and 5-year survival rate of 5 to $15 \% .^{1-4} \mathrm{GBC}$ is characterized by local invasion, extensive regional lymph node metastasis, vascular encasement and distant metastases, mode of spread are direct, lymphatic, vascular, neural, intraperitoneal and intraductal. ${ }^{5}$ Gallbladder carcinomas (T1 and T2) which are found incidentally in $0.2-2.9 \%$ after surgery for symptomatic gallstones, representing anywhere from 40 to $70 \%$ of all gallbladder cancer diagnoses. ${ }^{6,7}$ Gallbladder cancer is curable if treated at an early stage but notoriously difficult to cure, especially when it is associated with lymph node metastasis and/or liver metastasis. Because surgical resection represents the only modality offering potential cure, aggressive surgery is advocated..$^{2,3,7-10}$ It affects more females with a male to female ratio of 1:2 and commonly diagnosed in the seventh decade of life. ${ }^{7}$ Gallstones have been associated as a risk factor. ${ }^{11}$ Symptoms such as jaundice, palpable mass, changes in the bowel habit and ascites invariably represent the disease in the endstage with low curability. ${ }^{12}$ Lymphatic metastasis from gallbladder cancer spreads widely through hepatoduodenal ligament towards peripancreatic region and beyond strongly influenced by the depth of the primary tumor, so complete resection of the regional lymph nodes is important. ${ }^{3,13}$ Curative resection ranging from simple cholycystectomy to combined extended hepatectomy with common bile duct resection in the same stage. Radical surgery is advocated for patients in stages II and III. However considering the particularly poor prognosis of stage IV patients, radical resection appears less favorable than palliative resection in those who were complicated with hepatic invasion. ${ }^{14}$ Simple cholycystectomy is sufficient for T1a gallbladder cancer, but careful attention to the surgical margin of the cystic duct is essential because there is no difference in survival or recurrence rates between radical and simple cholycystectomy. ${ }^{7,11,15}$ Segment IVB 
and $\mathrm{V}$ hepatic resection and bile duct resection is usually indicated for $\mathrm{T} 2$ and /or T3 gallbladder cancer ${ }^{11,16}$ If the gallbladder cancer invades the hepatic hilar confluence, right hemihepatectomy or extended right hepatectomy should be considered. ${ }^{11,15-17}$ Hepatopancreaticoduodenectomy (HPD) has been performed to achieve radical resection. ${ }^{18}$ Organs in proximity such as the duodenum, the hepatic flexure of the colon and pancreas, bring up with technical challenges in achieving a negative margin and potentially curative resection. ${ }^{19}$ Gender, age, hepatic resection, and adjuvant chemotherapy did not influence postoperative survival. In contrast, tumor differentiation, hepatic invasion, choledochal invasion, lymph node metastasis, surgical margin status, and stage were significant predictors of survival of GBC patients. ${ }^{3,20}$

In the current study, we aimed to examine factors influencing the patient's survival following radical gall bladder cancer resection.

\section{Patients and methods}

\section{Patients' selections}

From January 2001 to December 2010 medical records of 38 patients underwent curative surgery for gall bladder carcinoma, who admitted to Surgical Oncology Department, South Egypt Cancer Institute (SECI), Assiut University, were retrospectively reviewed. Curative surgery was defined as complete resection of all macroscopic and microscopic tumors. Patients' demographic, clinical and diagnostic data were collected from chart review and office visits. Data included age, sex, clinical history, co-morbidity, preoperative investigation, as well as type of operation ,surgical morbidity and mortality, chemotherapy, radiotherapy, follow up data, survival, diagnostic procedures (ultrasonography, computed tomography (CT), magnetic resonance imaging (MRI),or magnetic resonance cholangiopancreatography (MRCP)) and tumor markers (CA19-9 \& CEA). Clinical staging of the disease was according to the American Joint Committee on Cancer (AJCC) staging system (7th edition). The criteria established in patients with GBC as an indication for radical resection at our institute comprise the absence of distant metastasis, peritoneal dissemination, liver or para-aortic lymph node metastasis, invasions on both sides of the portal vein or common hepatic artery, severe comorbidities or chronic liver disease. This study was conducted in accordance with the Helsinki Declaration and after the study had been approved by SECI Ethics Review Board. Median follow up period of the patients was 38 months (0.5-69 months).

\section{Surgical procedures}

Surgery for GBC ranged from simple cholycystectomy (whole gall bladder removed nothing else) for T1a tumor with negative cystic duct stump to radical cholycystectomy with partial resection of the adjacent liver tissue within $2.0 \mathrm{~cm}$ around the gallbladder bed/resection of segment $\mathrm{V}$ and IVB for T2 and/or T3 disease and with regional lymphadenectomy (enbloc clearance of cysticduct, pericholedochal, hepatic artery, portal vein, periduodenal and peripancreatic lymph nodes). Enbloc resection of adjacent organs was done if direct invasion occurred. Biliary continuity was restored by a Roux-en-Y biliary-enteric anastomosis when extra hepatic bile ducts were resected.

\section{Statistical analysis}

Univariate analysis for the most important factors regarding patients, tumor and operation carried out using chi-square and MannWhitney test analysis. Factors with proven statistical significance from the univariate analyses were further included in the multivariable Cox proportional hazard regression models. All non significant variables from the initial model were included in the final model one at a time to test for their effect on the model. Kaplan's Meier method with the logrank test for statistical significance was used for survival analysis of individual prognostic factors. All statistical analyses were performed using STATA v. 12 (STATA Corp., Texas, USA). A p-value $<0.05$ was considered significant.

\section{Results}

\section{Demographic and clinicopathological data}

During the study period of 10years, 38 patients with gall bladder cancer (GBC) underwent curative resection at Surgical Oncology Department, South Egypt Cancer Institute, Assiut University with median follow-up 38 months. The clinicopathological features were analyzed and summarized in (Table 1$) .16$ patients $(42.1 \%)$ were males and $22(57.9 \%)$ were females, with average age at presentation was 63 years old (38-86 years). 16 patients $(42.1 \%)$ had co-morbidities and Diabetes Mellitus had been the commonest (13.2\%).

Table I Demographic and clinicopathological data for patients presented with gall bladder cancer

\begin{tabular}{lll}
\hline Variables & Number & Percentage (\%) \\
\hline Age ( $\geq 75)$ & 21 & $54.60 \%$ \\
Gender (Female) & 22 & $57.90 \%$ \\
Co-Morbidity & 16 & $42.10 \%$ \\
Presentation & & \\
-Abdominal Pain & 29 & $75.40 \%$ \\
-Weight Loss & 20 & $52 \%$ \\
-Jaundice & 14 & $36.40 \%$ \\
-Abdominal Mass & 4 & $10.40 \%$ \\
-Gall Stones & 33 & $85.80 \%$ \\
Preoperative Biliary Drainage & 11 & $28.60 \%$ \\
Tumor Differentiation & & \\
-Well Differentiation & 15 & $51 \%$ \\
-Moderate Differentiation & 13 & $37 \%$ \\
-Poor Differentiation & 10 & $26 \%$ \\
Organ Invasion & 9 & $23.40 \%$ \\
Lymph vascular Invasion & 15 & $39 \%$ \\
Perineural Invasion & 11 & $8.60 \%$ \\
Tumor Size & & \\
TI/T2 & 12 & $31.20 \%$ \\
T3/4 & 26 & $67.60 \%$ \\
Co-Morbidity & & \\
Cardiac & 4 & $10.50 \%$ \\
Cirrhosis & $5.90 \%$ \\
COPD & 14 & $36.50 \%$ \\
Diabetes & $13.20 \%$ \\
Radiotherapy & & $68.40 \%$ \\
Chemotherapy & & \\
\hline & & \\
\hline
\end{tabular}

The most common symptom was pain in 29 patients $(75.4 \%)$ and the least common was abdominal mass. Most of the patients had a gall 
stones $(85.8 \%), 14$ patients presented with obstructive jaundice, and of those 11 patients needed preoperative biliary drainage. Fifty-two percent of patients presented with weight loss.

About two third (67.6\%) of the patients had a large tumor size (T3/ T4). The tumor histology was dominantly adenocarcinoma .Tumor differentiation was classified as well differentiated (51\%), moderately differentiated $(37 \%)$, and poorly differentiated $(26 \%)$. Perineural invasion was detected in only 2 cases, adjacent organ invasion was detected in 9 cases, and lymph vascular invasion was detected in 15 cases.

The surgical procedures, morbidity, mortality and recurrence for gall bladder cancer patients were illustrated in (Table 2). All patients underwent exploratory laparotomy for potentially curative resection and $\mathrm{R} 0$ resection (microscopic negative margin) performed. Radical cholecystectomy (RC) was performed in 34 patients while simple cholecystectomy (SC) was adequate in only 4 patients. In addition to radical cholecystectomy, concomitant resection of different nearby organs done in 23 patients, involving extended liver resection (ELR) in 3 patients, biliary tree resection in in 2 patients, duodenal resection in 4 patients, colonic resection in 2 patients and gastric resection only in 1 patient. Concurrent pancreatico-doudenectomy was performed in 2 patients due to doubtful distal biliary margins.

Table 2 Surgical procedures, morbidity, mortality and recurrence for gall bladder cancer patients

\begin{tabular}{lll}
\hline Surgical procedures & Frequency & Percent \\
\hline ELR & 3 & $7.90 \%$ \\
HPD & 2 & $5.30 \%$ \\
RC & 11 & $28.90 \%$ \\
RC + BD & 12 & $31.60 \%$ \\
RC + BD + Duodenal Sleeve Resection & I & $2.60 \%$ \\
RC + segmental Colonic Resection & I & $2.60 \%$ \\
RC + Gastric Sleeve Resection & 1 & $2.60 \%$ \\
RC + Sleeve Duodenectomy & 2 & $5.30 \%$ \\
RC + segmental Coloectomy + Duodenal Sleeve & 1 & $2.60 \%$ \\
SC & 4 & $10.50 \%$ \\
Total & 38 & $100.00 \%$ \\
Morbidity & & \\
-Liver Dysfunction & 2 & $5.20 \%$ \\
-Pneumonia & 3 & $7.80 \%$ \\
-Liver Abscess & I & $2.60 \%$ \\
-Bleeding & I & $2.60 \%$ \\
-Surgical Site Infection & 4 & $10.40 \%$ \\
-Dvt & 2 & $5.20 \%$ \\
-Biliary Leak & 1 & $2.60 \%$ \\
Total & 13 & $36.40 \%$ \\
Hospital Mortality & 13 & $2.60 \%$ \\
Tumor Recurrence & $37 \%$ \\
\hline & & \\
\hline
\end{tabular}

ELR, extended liver resection; HPD, hepatopancreatico-duodenectomy; RC, radical cholycystectomy; $\mathrm{BD}$, bile duct resection; $\mathrm{SC}$, simple cholycystectomy; DVT, deep venous thrombosis
Hospital mortality only occurred in 1 patient due to liver failure following extended liver resection. The morbidity rate in our study was $36.4 \%$. The general post-operative complications included pneumonia $(7.8 \%)$, surgical site infection $(10.4 \%)$ and deep venous thrombosis DVT (5.2\%). The specific complications included liver dysfunction $(5.2 \%)$, bleeding $(2.6 \%)$, liver abscess $(2.6 \%)$, and biliary leak $(2.6 \%)$.

Adjuvant chemotherapy was received in 26 patients $(67.6 \%)$ while 14 patients $(36.4 \%)$ received radiotherapy.

\section{Survival analysis}

First year overall survival estimate was $86.84 \% \pm 5.5 \%$, 2nd year $=$ $71.05 \% \pm 7.4 \%$, Third year $60.53 \% \pm 7.9 \%$ fifth year $=15.79 \% \pm 5.9 \%$ (Figure 1). Median DFS for GBC patients was 37 months.

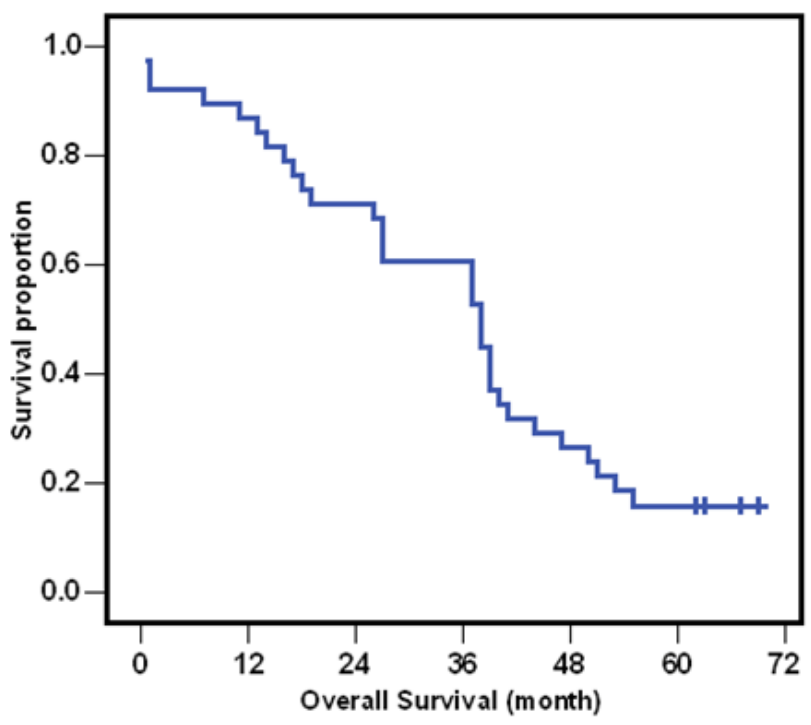

Figure I Overall survival of gall bladder cancer patients.

Factors found to have significance on survival of GBC patients upon univariate analysis underwent a multivariate analysis that revealed, tumor stage ( $p$ value $=0.004)$, lymph node metastasis $(p$ value $=0.03$ ) and both weight loss $(p$ value $=0.001)$ and jaundice $(p$ value $=0.03$ ) at presentation to be statistically significant.

Median survival for those having lymph node metastasis versus those not having lymph node metastasis was 18 months and 50 months , respectively $(\mathrm{p}=0.003)$.

Median survival for those presenting with weight loss and jaundice was 15 months and 17 months, with significant $\mathrm{p}$ values $(0.001$, $0.003)$, respectively.

Patients with early stages (stage I\&II) had better survival with median survival for those having early stages versus those having late stages (stage III\&IV) 53 months and 27 months, respectively $(\mathrm{p}=0.003)$.

Tumor recurrence occurred in 13 patients (34.2\%). The sites and nature of recurrence in these patients included peritoneal dissemination in 4 patients $(10.4 \%)$, liver metastases in 5 patients $(13 \%)$, lung metastases in 3 patients $(7.8 \%)$ and local recurrence in one patient $(2.6 \%)$ (Table 3$)$. 
Table 3 Univariate and multivariate analysis of predictors of overall survival of gall bladder cancer patients

\begin{tabular}{|c|c|c|}
\hline & $\begin{array}{l}\text { Univariate } \\
\text { analysis ( } P \text { value) }\end{array}$ & $\begin{array}{l}\text { Multivariate } \\
\text { analysis ( } P \text { Value) }\end{array}$ \\
\hline Age $(\geq 70 /<70)$ & 0.04 & 0.08 \\
\hline Gender (Female/Male) & 0.12 & \\
\hline Co-Morbidity & 0.03 & \\
\hline Abdominal Pain & 0.14 & \\
\hline Weight Loss & 0.0001 & 0.001 \\
\hline Jaundice & 0.005 & 0.03 \\
\hline Abdominal Mass & 0.066 & \\
\hline Gall Stones & 0.071 & \\
\hline $\begin{array}{l}\text { Tumor Differentiation } \\
\text { Wd-Md/Pd }\end{array}$ & 0.002 & 0.07 \\
\hline Organ Invasion & 0.004 & 0.06 \\
\hline Lymph Node Metastasis & 0.001 & 0.03 \\
\hline Perineural Invasion & 0.05 & \\
\hline $\begin{array}{l}\text { Tumor Size } \\
\text { TI-T2/T3-4 }\end{array}$ & 0.06 & \\
\hline $\begin{array}{l}\text { Tumor Stage } \\
\text { I -li /lii-lv }\end{array}$ & 0.003 & 0.004 \\
\hline Morbidity & 0.23 & \\
\hline Radiotherapy & 0.54 & \\
\hline Chemotherapy & 0.008 & \\
\hline
\end{tabular}

\section{Discussion}

Gallbladder cancer remains a disease with poor prognosis, with median DFS about 28 months. ${ }^{2,12,19}$ Non radical cholecystectomies had 5 -year overall survival rate of $17 \%$ reported by Wang et al, while the expected 5-year overall survival rates after curative resection was $40 \%{ }^{2,12,19,21}$ In our study the median DFS was 38 months with a 5-year survival rate of $16 \%$ after curative resection.

Gallbladder cancer has been reported as a disease of the elderly and females havea 40-60\% higher incidence than men(2). In our study, mean age at the time of diagnosis was 59years with female to male ratio of 1.4 Gallstones are a well-known risk factor for GBC. ${ }^{11,22,23}$ In this study, $86.8 \%$ of gallbladder patients had gall stones.

While jaundice is a poor prognostic factor in patients with gallbladder cancer, this does not necessarily preclude operative intervention as long as negative margins may be achieved. ${ }^{24,25}$ In our study, curative surgery achieved in 14 patients $(36.4 \%)$ presented with jaundice.

Although the rate of lymph node metastases has been reported to be less than $2.5 \%$ in stage Ia, survival was significantly impacted by the extent of surgery (LN dissection and RC), so cholecystectomy alone is inadequate in stage I GBC. ${ }^{26}$ In our study, $4(10.4 \%)$ patients with stage Ia GBC underwent simple cholycystectomy only with $100 \%$ 5-year overall survival rate. So simple cholycystectomy with negative margins is sufficient and these patients with stage Ia GBC do not need further surgical intervention and lymphadenectomy is generally not warranted.
Insufficient wedge resection of the liver result in a recurrence, while excessive extended right lobectomy may cause postoperative liver failure. ${ }^{27}$ In the current study, radical cholycystectomy was done in 29 patients (75.4\%) with partial resection of the adjacent liver tissue within $2 \mathrm{~cm}$ around the gallbladder bed and /or resection of segment $\mathrm{V}$ and $\mathrm{IVb}$ in T2 and /or T3 tumors. For selected patients, extended hepatectomy may be necessary to achieve negative margins and has been shown to be safe..$^{24,28}$ In our study extended liver resection (right hepaticectomy) was done only in 3 patients $(7.8 \%)$ due to gross liver invasion with reasonable morbidity.

Aggressive surgical resection including HPD was introduced to achieve curative surgical resection in patients with advanced GBC, although long-term survival was described by many surgeons but with high morbidity rates of 30.8- 91\% (hepatic failure occurred in $10-46.8 \%$ of patients) and mortality rates of $0-41.0 \%,{ }^{18,29,30}$ in current study, HPD was done in two patients $(5.2 \%)$ one of them died from hepatic failure, to prevent this lethal complication, many authors have recommended preoperative biliary drainage. ${ }^{12}$ In our study, preoperative biliary drainage was performed in 11 patients $(28.6 \%)$ presented with obstructive jaundice (9 patients with endoscopic retrograde biliary drainage and 2 patients with percutaneous transhepatic biliary drainage).

Routine extra hepatic bile duct resection does not increase the nodal yield or survival, but is associated with increased postoperative morbidity. Hence, it should be selectively performed to ensure negative surgical margins. ${ }^{12}$ In our study, resection of extra hepatic bile duct was selectively undertaken in patients with invasion of the bile duct by tumor (33.8\% of patients).

Lymph node involvement is a poor prognostic sign and nodalpositive patients with radical resection always show a better survival rate than nodal-positive patients without radical resection, stage for stage,,$^{24,31,32}$ in our study Lymph nodes negative patients $(47.4 \%)$ had median survival time of 50 months, while patients with positive lymph nodes metastasis $(52.6 \%)$ had median survival time 19 month ( $\mathrm{p}$ value $=0.001)$.

Higher T Stage, predict poor outcome in patients with gallbladder cancer. According to the depth of invasion, 5-years survival was $86 \%$ (T1), 56\% (T2), 45\% (T3), and 5\% (T4) $(10,12)$. Our study supports these findings as we detected significantly lower survival in stage (T3 and T4) disease, median survival was 27 months compared to 53 months for (T1 and T2).

Radical resection of GBC associated with a high morbidity (53\%) and mortality $(5 \%))^{33}$ In our study, postoperative complications occurred in $(36.4 \%)$ patients, and post operative mortality was seen in one patient $(2.6 \%)$, while 12 patients $(31.2 \%)$ died of recurrent disease. Para-aortic lymphadenectomy did not confer a survival benefit for patients with para-aortic disease, for whom the prognosis was as poor as that of distant metastases. ${ }^{8}$ Therefore, we did not perform formal para-aortic lymphadenectomy in patients with paraaortic lymph nodes metastasis.

Survival after surgical resection for patients with gallbladder carcinoma depends heavily on stage of disease. 5-year survival rate of patients with resected gallbladder carcinoma is $33-63 \%$ for patients at all disease stages and $9-20 \%$ for patients with stages III and IV (3). In our study 5 year survival was $16 \%$ this is may due to late presentation of most of our patients (stage III and IV 25 patients $(65.8 \%)$ and stage I and II 13 patients (34.2\%)). Patients with stage (I and II) had a median survival of 53 months while stage (III \& IV) patients had median survival of 27 months. 
Chemotherapy and radiation therapy are not effective in the treatment of gallbladder cancer, their survival benefits on patients with resected gallbladder carcinoma are controversial..$^{34}$ This is in agreement with our results which showed that, adjuvant chemotherapy was not received in 12 patients $(31.6 \%)$ and had median survival of 51 months, while 26 patients (68.4\%) received adjuvant chemotherapy and had median survival of 27 months with $p$ value $=0.0089$. Also, 24 patients $(63.2 \%)$ did not receive radiotherapy and had median survival of 39 months, while 14 patients (36.8\%) received radiotherapy and had median survival of 27 months with $P$ value $=0.5464$.

Tumor recurrence of GBC is high and chiefly intra-abdominal involving liver or retroperitoneal lymph nodes. ${ }^{12,35}$ In our study, tumor recurrence occurred in (34.2\%) of patients including peritoneal dissemination, liver metastases, lung metastases, and local recurrence.

The prognosis is poor for most patients of GBC with direct invasion of the liver and/or one other adjacent organ or structure. ${ }^{29}$ In current study, tumors with organ invasion had a median survival of 19 months compared to 39 months for patients with no organs invasion.

The limitations of this study were its retrospective design and the small number of patients studied. However, it did have few strong points as we studied a well annotated retrospective cohort of patients with clearly defined clinical, demographic, and pathologic variables along with distinct study outcomes. Hence, we recommend other studies on larger numbers of patients, including prospective studies to confirm the results of this study.

\section{Conclusion}

Curative surgical resection remains the only effective approach for treatment of GBC and can be done safely with a satisfying survival. This study confirms that jaundice, weight loss and aggressive tumor (advanced stage and regional lymph nodes metastasis) are poor prognostic factor. However, the presence of jaundice does not exclude resection, especially in highly selected patients.

\section{Acknowledgments}

None.

\section{Conflicts of interest}

The authors declare there is no conflict of interests.

\section{Funding}

None.

\section{References}

1. Aslam Ejaz, Teviah Sachs, Ihab R Kamel, et al. Gallbladder cancercurrent management options. Oncology \& Hematology Review. 2013;9(2):102-108.

2. Gourgiotis S, Kocher HM, Solaini L, et al. Gallbladder cancer. Am J Surg. 2008;196:252-264.

3. Lin HT, Liu GJ, Wu D, et al. Metastasis of primary gallbladder carcinoma in lymph node and liver. World J Gastroenterol. 2005;11(5):748-751.

4. Chapman BC, Jones T, McManus MC, et al. Metastatic papillary gallbladder carcinoma with a unique presentation and clinical course. JOP. 2014;15(5):515-559.

5. Einama T, Uchida K, Taniguchi M, et al. Successful curative resection of gallbladder cancer following S1 chemotherapy: A case report and review of the literature. Oncol Lett. 2014;8(6):2443-2447.
6. Sutariya VK, Patel PB, Tank AH. Port site and peritoneal metastases after laparoscopic cholecystectomy for incidentally found gallbladder carcinoma. Adv Biomed Res. 2013;2:39.

7. Lee HY, Kim YH, Jung GJ, et al. Prognostic factors for gallbladder cancer in the laparoscopy era. J Korean Surg Soc. 2012;83(4):227-236.

8. Nasu Y, Tanaka E, Hirano $\mathrm{S}$, et al. The prognosis after curative resection of gallbladder cancer with hilar invasion is similar to that of hilar cholangiocarcinoma. J Hepatobiliary Pancreat Sci. 2012;19:274-280.

9. Lin HT, Liu GJ, Wu D, et al. Metastasis of primary gallbladder carcinoma in lymph node and liver. World J Gastroenterol. 2005;11:748-751.

10. Kishi Y, Shimada K, Hata S, et al. Defination of T3/4 and Regional lymph Nodes in Gallbladder Cancer: Which Is More Valid, the UICC or the Japanese Staging System ?. Ann Surg Oncol. 2012;19:3567-3573.

11. Kai K, Aishima S, Miyazaki K. Gallbladder cancer: Clinical and pathological approach. World J Clin Cases. 2014;2(10):515-521.

12. Negi SS, Singh A, Chaudhary A. Lymph Nodal Involvement as Prognostic Factor in Gallbladder Cancer. Location,Count or Ratio?. J Gastrointest Surg. 2011;15:1017-1025.

13. Shimada H, Endo I, Togo S, et al. The role of lymph node dissection in the treatment of gallbladder carcinoma. Cancer. 1997;79:892-899.

14. Qu K, Chang HL, Liu SN, et al. Prognosis and management for gallbladder cancer with hepatic invasion: long-term results of 139 patients from a single center in China. Asian Pacific J Cancer Prev. 2012;13(3):1015-1018.

15. Araida T, Higuchi R, Hamano M, et al. Hepatic resection in 485 R0 pT2 and pT3 cases of advanced carcinoma of the gallbladder: results of a Japanese Society of Biliary Surgery survey--a multicenter study. $J$ Hepatobiliary Pancreat Surg. 2009;16:204-215.

16. Miyazaki M, Shimizu H, Ohtsuka M, et al. Hepatic S4a+S5 and bile duct resection for gallbladder carcinoma. $J$ Hepatobiliary Pancreat Sci. 2012;19(3):225-229.

17. Shimizu Y, Ohtsuka M, Ito H, et al. Should the extrahepatic bile duct be resected for locally advanced gallbladder cancer?. Surgery. 2004;136(5):1012-1017

18. Lim CS, Jang JY, Lee SE, et al. Reappraisal of hepatopancreatduodenectomy as a treatment modality for bile duct and gallbladder cancer. J Gastrointest Surg. 2012;16:1012-1018.

19. Dixon E, Vollmer CM Jr, Sahajpal A, et al. An aggressive surgical approach leads to improved survival in patients with gallbladder cancer : a 12-year study at a North American Center. Ann Surg. 2005;241(3):385394

20. Murakami Y, Uemura K, Sudo T, et al. Prognostic factors of patients with advanced gallbladder carcinoma following aggressive surgical resection. J Gastrointest Surg. 2011;15(6):1007-1016.

21. Wang SJ, Fuller CD, Kim JS, et al. Prediction model for estimating the survival benefit of adjuvant radiotherapy for gallbladder cancer. $J$ Clin Oncol. 2000;26:2112-2117.

22. Sheth S, Bedford A, Chopra S. Primary gallbladder cancer: recognition of risk factors and the role of prophylactic cholecystectomy. $\mathrm{Am} \mathrm{J}$ Gastroenterol. 2000;95:1402-1410.

23. Miyazaki M, Takada T, Miyakawa S, et al. Risk factors for biliary tract and ampullary carcinomas and prophylactic surgery for these factors. $J$ Hepatobiliary Pancreat Surg. 2008;15(1):15-24.

24. Yildirim E, Celen O, Gulben $\mathrm{K}$, et al. The surgical management of incidental gallbladder carcinoma. Eur J Surg Oncol. 2005;31(1):45-52.

25. Regimbeau JM, Fuks D, Bachellier P, et al. Prognostic value of jaundice in patients with gallbladder cancer by the AFCGBC- 2009 study group. Eur J Surg Oncol. 2011;37(6):505-512. 
26. Hari DM, Howard JH, Leung AM, et al. A 21-year analysis of stage I gallbladder carcinoma: is cholecystectomy alone adequate? HPB (Oxford). 2013;15(1):40-48.

27. Tsuji T, Kanemitsu K, Hiraoka T, et al. A new method to establish the rational extent of hepatic resection for advanced gallbladder cancer using dye injection through the cystic artery. HPB (oxford). 2004;6(1):33-66.

28. Reddy SK, Marroquin CE, Kuo PC, et al. Extended hepatic resection for gallbladder cancer. Am J Surg. 2007;194(3):355-361.

29. Ebata T, Nagino $M$, Nishio $H$, et al. Right hepatopancreatoduodenectomy: improvements over 23 years to obtain acceptability. $J$ Hepatobiliary Pancreat surg. 2007;14(2):131-135.

30. Nimura Y, Kamiya J, Kondo S, et al. Aggressive preoperative management and extended surgery for hilar cholangiocarcinoma: Nagoya experience. J Hepatobiliary Pancreat surg. 2000;7(2):155-162.

31. Goetze TO, Paolucci V. The prognostic impact of positive lymph nodes in stages T1 to T3 incidental gallbladder carcinoma: results of the German Registry. Surg Endosc. 2012;26(5):1382-1389.
32. Zaydfudim V, Feurer ID, Wright JK, et al. The impact of tumor extent ( $\mathrm{T}$ stage) and lymph node involvement ( $\mathrm{N}$ stage) on survival after surgical resection for gallbladder adenocarcinoma. HPB (Oxford). 2008; $10(6): 420-427$.

33. Burdiles P, Csendes A, Diaz JC, et al. Factors affecting mortality in patients over 70 years of age submitted to surgery for gallbladder or common bile duct stones. Hepatogastroenterology. 1989;36(3):136139.

34. Nishio H, Nagino M, Ebata T, et al. Aggressive surgery for stage 4 gallbladder carcinoma, what are the contraindications?. J Hepatobiliary Pancreatic Surg. 2007;14(4):351-357.

35. Theodoros EP, Efstathios TP, Nikolaos GS, et al. Current curative surgical management of gallbladder cancer-a brief review. Journal of current surgery. 2012;2(3):10.4021/jcs50w. 